\title{
An algebraic proof of Zak's inequality for the dimension of the Gauss image
}

\author{
Aron Simis ${ }^{1, \star}$, Karen E. Smith ${ }^{2, \star \star}$, Bernd Ulrich ${ }^{3, \star \star \star}$ \\ 1 Universidade Federal de Pernambuco, 50740-540 Recife, PE, Brazil \\ 2 University of Michigan, Ann Arbor, MI 48109, USA \\ 3 Purdue University, West Lafayette, IN 47907, USA
}

Received: 12 April 2001; in final form: 31 January 2002 /

Published online: 8 November 2002 - (c) Springer-Verlag 2002

\section{Introduction}

A classical theorem in complex algebraic geometry states that, for any smooth projective variety, the Gauss map is finite; in particular, a smooth variety and its Gauss image have the same dimension (with the obvious exception of a linear space). Furthermore, even when the variety is not smooth, Zak proved a lower bound on the dimension of its Gauss image in terms of the dimension of its singular locus. Our purpose in this note is to reinterpret Gauss maps within a more general algebraic framework, and thus recover Zak's bound on the dimension of the Gauss image as a special case of an interesting new bound on the analytic spread of a module of Kähler differentials. This connects that classical subject in complex geometry to recent research in commutative algebra concerning integral closures of modules. In particular, we give a new, purely algebraic proof of Zak's theorem.

We recall a precise version of Zak's theorem. Let $X$ be an irreducible projective variety of dimension $d$ defined over an algebraically closed field $k$, considered with a fixed embedding $X \subset \mathbb{P}(V)$ for some finite dimensional $k$-vector space $V$. The Gauss map is the rational map from $X$ to the Grassmannian of projective $d$-planes in $\mathbb{P}(V)$ assigning to each smooth $k$-point of $X$ the projective tangent plane there,

$$
\Gamma: X \rightarrow \mathbb{G}(\operatorname{dim} X, \mathbb{P}(V))
$$

\footnotetext{
* Partially supported by CNPq, Brazil.

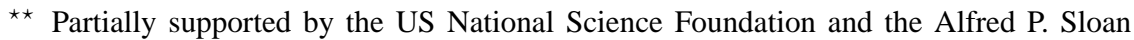
Foundation.

$\star \star \star$ Partially supported by the US National Science Foundation.
} 


$$
p \mapsto T_{p} X .
$$

The Gauss image of $X$, denoted $\Gamma(X)$, is the closure of the image of the smooth locus of $X$ under $\Gamma$. Zak's theorem states that, provided $X$ is not a linear subvariety of $\mathbb{P}(V)$, the dimension of the Gauss image satisfies the inequality

$$
\operatorname{dim} \Gamma(X) \geq \operatorname{dim} X-\operatorname{dim} \operatorname{Sing}(X)-1,
$$

where $\operatorname{Sing}(X)$ denotes the closed locus of non-smooth points of $X$ and $\operatorname{dim} \emptyset=-1[Z, 2.8$, p.23]. In the smooth case one obtains $\operatorname{dim} \Gamma(X)=$ $\operatorname{dim} X$, a result that was known before, at least for complex algebraic varieties ([GH, 2.29]).

In this paper, we deduce Zak's theorem from our main result, which is an interesting bound on the analytic spread of a module of Kähler differentials. The analytic spread is an important invariant in the theory of integral closures of ideals - and more recently modules - over a commutative local ring. Roughly, the analytic spread of an ideal $I$ in a local ring $R$ is the smallest number of generators of any ideal having the same integral closure as $I$; more geometrically it can be formulated in terms of the dimension of the closed fiber under the blowing up morphism of the scheme $\operatorname{Spec}(R)$ along $V(I)$. The notions of analytic spread and integral closure are well-understood for ideals; the natural extension of these notions to modules is of more recent interest. The precise definitions are recalled in Sect. 2.

The main objective of this work is to give a lower bound for the analytic spread of $\bigwedge^{d} \Omega_{R / k}$ in terms of the singular locus of $\operatorname{Spec}(R)$, where $\Omega_{R / k}$ denotes the module of Kähler $k$-differentials on a standard graded domain $R$ over a field $k$, and $d$ denotes the dimension of $R$. Specifically, Theorem 2.1 establishes that unless $R$ is a polynomial ring, this analytic spread is at least as large as the codimension of the locus of non-smooth points of $R$ over $k$. The fundamental importance of this result is evidenced by the fact that it quickly recovers Zak's bound on the dimension of the Gauss image; indeed, one might call it an algebraic interpretation of Zak's bound.

Our method for bounding the analytic spread of $\bigwedge^{d} \Omega_{R / k}$ is to use the so-called canonical class $\mathfrak{c}_{R / k}: \bigwedge^{d} \Omega_{R / k} \rightarrow \omega_{R / k}$, a natural map from the module of differential $d$-forms to the graded canonical module of $R$. We show that the required bound holds provided $\mathfrak{c}_{R / k}$ is not integral, that is, provided that the inclusion

$$
\mathfrak{c}_{R / k}\left(\bigwedge^{d} \Omega_{R / k}\right) \subset \omega_{R / k}
$$

is not an integral extension of modules in the sense discussed in Sect. 2. An essential point of our argument is that the singularity of $\operatorname{Spec}(R)$ forces this extension to be non-integral. To see this, we argue in terms of the 
homogeneous pieces of $\omega_{R / k}$ : noting that an integral extension of $\bigwedge^{d} \Omega_{R / k}$ can contain no element of degree less than $d$, we then show by contrast that $\omega_{R / k}$ must contain an element of degree $d-1$. This result, which may be of independent interest, is stated in a dual form in Theorem 2.3: the $a$-invariant of $R$ is greater than $-d$ unless $R$ is a polynomial ring. To fetch a nonzero element of $H_{\mathfrak{m}}^{d}(R)$ of degree greater than $-d$ the theory of tight closure is used.

In Sect. 3 of the paper, we show how our bound on the analytic spread of the module of Kähler differentials leads to the classical theorem on the Gauss map. For this, we develop more generally a notion of algebraic "Gauss maps" attached to a finitely generated graded $R$-module $M$ that, when applied to the case where $R$ is the homogeneous coordinate ring of $X$ in $\mathbb{P}(V)$ and $M=\Omega_{R / k}$, leads to the classical Gauss map for the projective variety $X$. The connection to the module $\bigwedge^{d} \Omega_{R / k}$ is via the Plücker embedding. Zak's theorem then becomes an immediate corollary of the main bound in Theorem 2.1.

Our estimate leads naturally to a more general question. To wit, let $M$ be a finitely generated graded module of rank $r$ (which is not a direct sum of a free module and a torsion module) over a standard graded domain. When is the analytic spread of $\bigwedge^{r} M$ at least as large as the codimension of the closed locus of $\operatorname{Spec}(R)$ where $M$ is not free?

\section{The main theorem}

If $M$ is a finitely generated module over a Noetherian domain $R$, then the Rees algebra $\mathcal{R}(\mathcal{M})$ is the symmetric algebra of $M$ modulo the ideal of $R$-torsion elements. If $M=I$ is an ideal in $R$, then of course $\mathcal{R}(\mathcal{I})$ is the classical Rees algebra

$$
R \oplus I \oplus I^{2} \oplus \ldots
$$

whose associated projective scheme $\operatorname{Proj}(\mathcal{R}(\mathcal{I}))$ defines the blowup of $\operatorname{Spec}(R)$ along $V(I)$.

Given a map $N \longrightarrow M$ of finitely generated $R$-modules, there is of course an induced map

$$
\mathcal{R}(\mathcal{N}) \longrightarrow \mathcal{R}(\mathcal{M})
$$

of Rees algebras. We say that the map $N \longrightarrow M$ is integral if the induced map of Rees algebras is finite, that is, if $\mathcal{R}(\mathcal{M})$ is finitely generated as a module over $\mathcal{R}(\mathcal{N})$. If the map $N \hookrightarrow M$ is an inclusion, we also say that the module $M$ is integral over $N$. For an inclusion of ideals $J \subset I$, this notion recovers the standard notion of integral dependence, which is commonly defined as follows: each element $z$ of $I$ satisfies some polynomial

$$
z^{n}+a_{1} z^{n-1}+\ldots+a_{n}=0
$$


where $a_{i} \in J^{i}$ for all $i$.

If $M$ is a finitely generated module over a local domain $R$, then the analytic spread $\ell(M)$ is defined to be the dimension of the fiber ring

$$
\mathcal{R}(M) \otimes_{R} R / \mathfrak{m}
$$

where $\mathfrak{m}$ is the unique maximal ideal of $R$. When $M=I$ is an ideal of $R$, note that the projective scheme $\operatorname{Proj}(\mathcal{R}(M)) \otimes_{R} R / \mathfrak{m}$ is precisely the scheme-theoretic fiber over the closed point $\mathfrak{m}$ in $\operatorname{Spec}(R)$ under the blowing up morphism Proj $(\mathcal{R}(I)) \longrightarrow \operatorname{Spec}(R)$. Thus the analytic spread is one more than the dimension of this closed fiber. If the residue field $R / \mathfrak{m}$ is infinite, then the analytic spread is the smallest possible minimal number of generators for an ideal over which $I$ is integral. When $M$ is a finitely generated graded module over a finitely generated $\mathbb{N}$-graded ring $R$ over a field $k=R_{0}$, the analytic spread is defined analogously, with $\mathfrak{m}$ denoting the unique homogeneous maximal ideal of $R$. For generalities on Rees rings of modules, see [SUV].

Our main result reads as follows.

Theorem 2.1. Let $R$ be a standard graded domain of dimension d. Assume that $R_{0}=k$ is a field algebraically closed in the quotient field of $R$. If $R$ is not a polynomial ring, then the analytic spread of the module of differential $d$-forms of $R$ is at least the codimension of the locus of non $k$-smooth points of $R$, that is,

$$
\ell\left(\bigwedge^{d} \Omega_{R / k}\right) \geq \operatorname{codim} \operatorname{Sing}(R) .
$$

By standard graded, we mean that $R$ is an $\mathbb{N}$-graded algebra, finitely generated by its degree one elements over its degree zero component $R_{0}=k$.

In order to prove this result we develop some preliminaries. Let, more generally, $R$ be a Noetherian $\mathbb{N}$-graded domain of dimension $d$. Assume that $R_{0}=k$ is an infinite field and that the quotient field $L$ of $R$ is separable over $k$. Consider the graded canonical module $\omega_{R / k}$ of $R$, which is the graded $k$-dual of $H_{\mathfrak{m}}^{d}(R)$, the highest local cohomology module of $R$ with support in its homogeneous maximal ideal. According to $[\mathrm{E}],[\mathrm{L}]$ or $[\mathrm{KW}]$, there exists a homogeneous $R$-linear map

$$
\mathfrak{c}_{R / k}: \bigwedge^{d} \Omega_{R / k} \rightarrow \omega_{R / k},
$$

that is an isomorphism locally on the smooth locus of $R$. This map is called the canonical class of $R$. In fact, $\mathfrak{c}_{R / k}$ can be defined by taking $\omega_{R / k} \subset$ $\bigwedge^{d} \Omega_{L / k}$ as the Dedekind complementary module of $R$ and showing that this module contains the image of the localization map $\bigwedge^{d} \Omega_{R / k} \rightarrow \bigwedge^{d} \Omega_{L / k}$. 
Proposition 2.2. Let $R$ be a Noetherian $\mathbb{N}$-graded domain of dimension $d$. Assume that $R_{0}=k$ is an infinite field and that the quotient field of $R$ is separable over $k$. If $\ell\left(\bigwedge^{d} \Omega_{R / k}\right)<\operatorname{codim} \operatorname{Sing}(R)$, then the map $\mathfrak{c}_{R / k}$ is integral.

Proof. Assume that $\ell\left(\bigwedge^{d} \Omega_{R / k}\right)<s$ where $s$ is the codimension of the non-smooth locus of $R$. Multiplying the inclusion $\operatorname{im}\left(\mathfrak{c}_{R / k}\right) \subset \omega_{R / k}$ by a fixed nonzero homogeneous element of $R$, we get an inclusion $J \subset I$ of homogeneous ideals of $R$. In this setup one has to prove that $I \subset \bar{J}$, where $\bar{J}$ denotes the integral closure of $J$. Clearly, it is enough to verify the latter inclusion after localization at each associated prime of $R / \bar{J}$. Since $R$ is universally catenary, a result due to McAdam [M, 4.1] shows that

$$
\operatorname{dim} R_{P} \leq \ell(\bar{J})=\ell(J)
$$

for any prime $P$ associated to $R / \bar{J}$. Since $\ell\left(\bigwedge^{d} \Omega_{R / k}\right)<s$ by our assumption, we know that $\ell(J)<s$, so that for any prime $P$ associated to $R / \bar{J}$, we have the inequality $\operatorname{dim} R_{P}<s$. This forces $P$ to be in the smooth locus of $\operatorname{Spec}(R)$. Thus, as seen above, $J_{P}=I_{P}$. It follows that $I R_{P} \subset \bar{J} R_{P}$ for every prime ideal $P$ associated to $R / \bar{J}$, and the proposition is proved.

Proof of Theorem 2.1. Replacing $k$ by $k(t)$, where $t$ is an indeterminate, we may assume that $k$ is infinite. We may further suppose that codim $\operatorname{Sing}(R)>0$ in which case the quotient field of $R$ is separable over $k$ and $R$ is geometrically reduced. According to Proposition 2.2, it suffices to show that the canonical class

$$
\mathfrak{c}_{R / k}: \bigwedge^{d} \Omega_{R / k} \rightarrow \omega_{R / k}
$$

is not integral unless $R$ is a polynomial ring. To do this, we make use of the following easy-to-prove fact: if $N \subset M$ is an integral extension of torsionfree finitely generated graded modules over a graded domain $R$, then no element of $M$ can have degree less than the smallest non-zero degree that occurs in $N$.

Recall that the $a$-invariant of $R$ can be defined as

$$
a(R)=-\min \left\{n \in \mathbb{Z} \mid\left[\omega_{R / k}\right]_{n} \neq 0\right\}
$$

where $\left[\omega_{R / k}\right]_{n}$ denotes the $n$-th graded part of $\omega_{R / k}$. If $a(R)>-d$, then $\omega_{R / k}$ has nontrivial elements of degree less than $d$. On the other hand, because $R$ is generated by elements of degree one, the module $\wedge^{d} \Omega_{R / k}$ is generated in degree $d$, as the elements $d x_{1} \wedge \ldots \wedge d x_{d}$ generate it for $x_{1}, \ldots, x_{d}$ ranging through all the degree one algebra generators of $R$. So 
because the map $\mathfrak{c}_{R / k}$ is degree preserving, the extension of torsion-free modules

$$
\mathfrak{c}_{R / k}\left(\bigwedge^{d} \Omega_{R / k}\right) \subset \omega_{R / k}
$$

cannot be integral when $a(R)>-d$. Thus Theorem 2.1 follows from the next theorem.

Theorem 2.3. Let $R$ be a standard graded domain of dimension d. Assume that $R_{0}=k$ is a field algebraically closed in the quotient field of $R$, and that $R$ is geometrically reduced. If $R$ is not a polynomial ring, then the $a$-invariant of $R$ satisfies $a(R)>-d$.

Proof. The module $\omega_{R / k}$ is the graded $k$-dual of $H_{\mathfrak{m}}^{d}(R)$, where $\mathfrak{m}$ denotes the homogeneous maximal ideal of $R$. Thus it suffices to show that $H_{\mathfrak{m}}^{d}(R)$ has a nonzero element of degree $-d+1$. We may assume that $k$ is infinite, in which case $R$ admits a homogeneous system of parameters $x_{1}, \ldots, x_{d}$ of degree one.

Recall that $H_{\mathfrak{m}}^{d}(R)$ can be identified with the cokernel of the map

$$
\begin{aligned}
R_{x / x_{1}} \oplus \ldots \oplus R_{x / x_{d}} & \longrightarrow R_{x} \\
\left(\frac{s_{1} x_{1}^{t}}{x^{t}}, \ldots, \frac{s_{d} x_{d}^{t}}{x^{t}}\right) & \mapsto \frac{\sum_{i=1}^{d}(-1)^{i} s_{i} x_{i}^{t}}{x^{t}}
\end{aligned}
$$

where $x=x_{1} \cdots x_{d}$. Thus we represent an element $\eta$ of $H_{\mathfrak{m}}^{d}(R)$ by an equivalence class of fractions

$$
\eta=\left[\frac{w}{x^{t}}\right] .
$$

It is not difficult to check that the element $\eta$ is zero if and only if there exists a natural number $s$ such that $x^{s} w \in\left(x_{1}^{t+s}, \ldots, x_{d}^{t+s}\right)$. Now, provided that $R$ is not regular, the maximal ideal $\mathfrak{m}$ is not generated by $x_{1}, \ldots, x_{d}$, so we can find an element $z$ of degree one not in the ideal $\left(x_{1}, \ldots, x_{d}\right)$. Consider the element

$$
\eta=\left[\frac{z}{x}\right]
$$

in $H_{\mathfrak{m}}^{d}(R)$. This element has degree $-d+1$, provided it is nonzero. So we need only show that $\eta \neq 0$.

Supposing to the contrary, there exists $s$ such that $x^{s} z \in$ $\left(x_{1}^{s+1}, \ldots, x_{d}^{s+1}\right) R$. By the colon-capturing property of tight closure ([HH1, 7.9], [HH3, 4.1.7]) this implies that $z$ belongs to the tight closure $\left(x_{1}, \ldots, x_{d}\right)^{*}$ of $\left(x_{1}, \ldots, x_{d}\right)$. But the tight closure of an ideal generated 
by elements of a fixed degree $e$ in a ring satisfying the hypothesis of Theorem 2.3 contains no element of degree $e$ not already in the ideal ([S2, 2.4]). So $z \in\left(x_{1}, \ldots, x_{d}\right)$, contrary to our choice of $z$. This contradiction establishes that $\eta=\left[\frac{z}{x}\right]$ is a nonzero element of degree $-d+1$, and the theorem is proved.

Remark 2.4. Here is an alternate proof of Theorem 2.3, perhaps more intuitive, in the case where $k$ is an algebraically closed field of positive characteristic. Note that if $x_{1}, \ldots, x_{d}$ form a regular sequence on $R$, then $\eta=\left[\frac{z}{x}\right]$ is zero if and only if $z \in\left(x_{1}, \ldots, x_{d}\right)$. Now if $\eta$ is zero in $H_{\mathfrak{m}}^{d}(R)$, its image in $H_{\mathfrak{m}}^{d}\left(R^{+g r}\right)$ is also zero, where $R^{+g r}$ is a graded absolute integral closure of $R$, that is, the subring of the integral closure of $R$ in an algebraic closure of its quotient field generated by homogeneous elements of integer degree. Since $R^{+g r}$ is a Cohen-Macaulay $R$-algebra ([HH2, 5.15]), if $\eta=\left[\frac{z}{x}\right] \in H_{\mathfrak{m}}^{d}\left(R^{+g r}\right)$ is zero, then $z \in\left(x_{1}, \ldots, x_{d}\right) R^{+g r}$. Since $z \in[R]_{1}$ and $\left[R^{+g r}\right]_{0}=\bar{k}=k$, it follows that $z \in\left(x_{1}, \ldots, x_{d}\right) R$, and we arrive at the same contradiction as in the above proof of Theorem 2.3.

Actually, the argument is essentially the same as the original. Indeed, for any ideal $I$ generated by a homogeneous system of parameters in a graded domain of prime characteristic, it is known that $I^{*}=I R^{+g r} \cap R$ ([S1, Theorem 1]). We can use the above alternative, more generally, by using reduction to prime characteristic, and then passing to the case where the base field is algebraically closed. These steps are somewhat technical, but of course our proof above hides the technical difficulties behind the definition of tight closure in characteristic zero.

\section{Algebraic "Gauss maps"}

In this section we develop a general algebraic theory of Gauss maps, which will allow us to deduce Zak's bound on the dimension of the Gauss image of a projective variety as a corollary of the main theorem of the preceding section.

Let $R$ denote a standard graded domain with $R_{0}=k$ an algebraically closed field, and let $\mathfrak{m}$ be the homogeneous maximal ideal of $R$. Write $X=$ $\operatorname{Proj}(R)$. Thinking of $X$ in this way, we have essentially fixed an embedding $X \subset \mathbb{P}(V)$ where $V=R_{1}^{*}$ is the $k$-vector space of linear functionals on the space of degree one forms in $R$. Let $M$ be any finitely generated graded $R$-module of rank $r$ generated by homogeneous elements of the same degree, and let $F$ be a graded free $R$-module generated by a finite set of homogeneous elements of the same degree mapping surjectively onto $M$ by a degree-preserving map. Write $W$ for the $k$-vector space $\left(F \otimes_{R} R / \mathfrak{m}\right)^{*}$, the $k$-dual of $F \otimes_{R} R / \mathfrak{m}$. 
For any closed $k$-point $p=\left[a_{0}, \ldots, a_{n}\right] \in X \subset \mathbb{P}(V)$, pick a maximal ideal $\mathfrak{m}_{a}:=\left(x_{0}-a_{0}, \ldots, x_{n}-a_{n}\right)$ of $R$ corresponding to a representative of $p$ on the affine cone over $X$ in $V$. Note that there is a natural isomorphism of vector spaces $F \otimes_{R} R / \mathfrak{m}_{a} \simeq W^{*}$. Thus the surjection $F \rightarrow M$ induces a $k$-linear surjection $W^{*} \rightarrow M \otimes_{R} R / \mathfrak{m}_{a}:=M_{a}$ and therefore an embedding of $k$-vector spaces

$$
M_{a}^{*} \hookrightarrow W .
$$

The image $M_{a}^{*}$ in $W$ depends only on $p$, and for any $p$ in the free locus of $M$, is a $k$-vector space of dimension $r$. This yields a well-defined map on the free locus of the module $M$,

$$
\gamma_{M}: X \rightarrow \mathbb{G}(r-1, \mathbb{P}(W)) \subset \mathbb{P}\left(\bigwedge^{r} W\right),
$$

where the last inclusion is the Plücker embedding of $\mathbb{G}(r-1, \mathbb{P}(W))$. In the case where $M=\Omega_{R / k}$, one recovers the ordinary Gauss map of $X \subset \mathbb{P}(V)$, by identifying $W$ with $V$. The following result shows that $\gamma_{M}$ is indeed a rational map on $X$ and, furthermore, identifies the coordinate ring of its image in $\mathbb{P}\left(\bigwedge^{r} W\right)$. (By definition, the image of a rational map $\gamma$ is the closure of the image of $\gamma$ restricted to a dense open set where $\gamma$ is regular.)

Proposition 3.1. With notation as in the preceding paragraph, $\gamma_{M}$ is a rational map on $X$ and the homogeneous coordinate ring of the image of $\gamma_{M}$ in $\mathbb{P}\left(\bigwedge^{r} W\right)$ is

$$
\mathcal{R}\left(\bigwedge^{r} M\right) \otimes_{R} R / \mathfrak{m}
$$

Proof. Consider the exact sequence of graded modules $0 \rightarrow U \rightarrow F \rightarrow$ $M \rightarrow 0$ where $F$ is a free module generated by finitely many homogeneous elements of the same degree. There exists a homogeneous free $R$-submodule $U^{\prime}$ of $U$ which coincides with $U$ at the minimal prime of $R$, and hence generically on $X$. Let $M^{\prime}=F / U^{\prime}$. The natural surjection $M^{\prime}=F / U^{\prime} \rightarrow$ $F / U \simeq M$ is obtained by factoring out a torsion $R$-module, so similarly $\bigwedge^{r} M^{\prime}$ surjects onto $\bigwedge^{r} M$ with an $R$-torsion kernel, and the natural map of Rees rings $\mathcal{R}\left(\bigwedge^{r} M^{\prime}\right) \rightarrow \mathcal{R}\left(\bigwedge^{r} M\right)$ is an isomorphism. Thus, without loss of generality, we may replace $M$ by $M^{\prime}$ and therefore assume that $M$ has projective dimension one, i.e., that $U$ is free. Let $c$ be the rank of $U$.

Using the natural identification of the set of $r$-dimensional subspaces of the vector space $W$ with the set of complementary-dimensional subspaces of $W^{*}$, we can interpret the map $\gamma_{M}$ as a map $X \stackrel{{ }^{\epsilon_{M}} \rightarrow}{\rightarrow} \mathbb{P}\left(\bigwedge^{c} W^{*}\right)$ sending $p$ to the image of the $k$-linear map $U \otimes_{R} R / \mathfrak{m}_{a} \rightarrow F \otimes_{R} R / \mathfrak{m}_{a}=W^{*}$. It suffices to show that $\epsilon_{M}$ is a rational map and that the coordinate ring of the image of $\epsilon_{M}$ is isomorphic to $\mathcal{R}\left(\bigwedge^{r} M\right) \otimes_{R} R / \mathfrak{m}$. Now fix homogeneous 
generators for $U$ and homogeneous generators $e_{0}, \ldots, e_{n}$ for $F$, and let $\phi$ be the matrix corresponding to the presentation map $U \rightarrow F$ in these bases. Let $\Delta_{j_{1} \ldots j_{c}}$ be the maximal minor of $\phi$ indexed by the rows $j_{1}, \ldots, j_{c}$. Then the map $\epsilon_{M}$ described above sends a point $p \in X$ to the point $\left[\Delta_{j_{1} \ldots j_{c}}(p)\right]$ of $\mathbb{P}\left(\bigwedge^{c} W^{*}\right)=\mathbb{P}\left(\oplus k e_{j_{1}} \wedge \ldots \wedge e_{j_{c}}\right)$. This shows that $\epsilon_{M}$ is a rational map which is regular on the locus where $\phi$ has full rank (the free locus of $M$ ). Furthermore, if $I$ denotes the ideal of maximal minors of $\phi$, then $\mathcal{R}(I)$ is the bigraded coordinate ring of the graph of $\epsilon$ considered as a subscheme of $\mathbb{P}(V) \times \mathbb{P}\left(\bigwedge^{c} W^{*}\right)$. Thus $\mathcal{R}(I) \otimes_{R} R / \mathfrak{m}$ is the homogeneous coordinate ring of the projection of the graph onto $\mathbb{P}\left(\bigwedge^{c} W^{*}\right)$, which is to say, the image of $\epsilon$.

Thus it remains only to show that $\mathcal{R}(I)=\mathcal{R}\left(\bigwedge^{r} M\right)$. The map $U \stackrel{\phi}{\rightarrow} F$ induces a map

$$
\bigwedge^{r} F \simeq\left(\bigwedge^{c} F\right)^{\vee} \stackrel{\left(\wedge^{c} \phi\right)^{\vee}}{\longrightarrow}\left(\bigwedge^{c} U\right)^{\vee} \simeq R
$$

whose image is $I$, where $-^{\vee}=\operatorname{Hom}(-, R)$. This map factors through $\bigwedge^{r} M$, and thus induces a surjection $\bigwedge^{r} M \rightarrow I$. Because $\bigwedge^{r} M$ has rank one, the kernel is torsion, so $\mathcal{R}\left(\bigwedge^{r} M\right) \simeq \mathcal{R}(I)$.

As a corollary to Proposition 3.1, we obtain the following result.

Corollary 3.2. Let $X$ be a reduced and irreducible variety of dimension $d-1$ over an algebraically closed field $k$ and fix an embedding $X \subset \mathbb{P}(V)$, where $V$ is a finite dimensional $k$-vector space. Let $R$ be the homogeneous coordinate ring of this embedding and let $\mathfrak{m}$ denote its homogeneous maximal ideal. Then the homogeneous coordinate ring of the Gauss image in the Plücker embedding $\Gamma(X) \subset \mathbb{P}\left(\bigwedge^{d} V\right)$ is

$$
\mathcal{R}\left(\wedge^{d} \Omega_{R / k}\right) \otimes_{R} R / \mathfrak{m} .
$$

It is now clear that Zak's inequality for the dimension of the Gauss image of $X$ is an immediate consequence of Theorem 2.1 and Corollary 3.2.

Remark 3.3. It is of course also possible to study a "local Gauss mapping" and ask whether Theorem 2.1 holds more generally. Let $(R, \mathfrak{m})$ be a Noetherian local domain essentially of finite type over an algebraically closed field $k$, and set $d=\operatorname{dim} R+\operatorname{trdeg}_{k} R / \mathfrak{m}$. A local version of Zak's theorem would predict that the analytic spread of $\bigwedge^{d} \Omega_{R / k}$ is greater than or equal to the codimension of the singular locus of $R$ (provided that $R$ is not regular). Does this hold true? In other words, is $\ell\left(\bigwedge^{d} \Omega_{R / k}\right) \geq$ height $F_{d}$, where $F_{d}$ is the $d$-th Fitting ideal of $\Omega_{R / k}$ ? 
One approach to this question is to try to carry out the same sort of argument we used to settle the graded case. One still has the canonical class

$$
\mathfrak{c}_{R / k}: \bigwedge^{d} \Omega_{R / k} \rightarrow \omega_{R / k}
$$

that is an isomorphism locally on the smooth locus of $R$. We ask: If $R$ is not regular, is it true that $\mathfrak{c}_{R / k}$ is not integral? Proposition 2.2 can be adapted to this setting, so a positive answer to this question would establish the local version of Zak's theorem proposed above. In addition to our work here in the graded case, there is some evidence to support this approach.

For example, assuming $R$ is Cohen-Macaulay, a positive answer follows from work of Kunz and Waldi ([KW, 5.20]). Also, a positive answer can be shown in the quasi-Gorenstein case (that is, when $\omega_{R / k}$ is free), using the Dedekind different and the theorem on the purity on the branch locus. It seems reasonable to conjecture that in the general setting described above, $\mathfrak{c}_{R / k}$ is never integral unless $R$ is regular.

Remark 3.4. It is natural to investigate whether the local version of Zak's theorem might hold much more generally. Namely, if $R$ is a Noetherian local domain and $M$ is a finitely generated $R$-module of rank $r$ (which is not a direct sum of a free module and a torsion module), when is the analytic spread of $\bigwedge^{r} M$ at least the codimension of the non-free locus of $M$ ? In other words, when is $\ell\left(\bigwedge^{r} M\right) \geq$ height $F_{r}(M)$, where $F_{r}(M)$ is the $r$-th Fitting ideal of $M$ ? In light of Proposition 3.1, this inequality in the graded case implies a general statement analogous to Zak's theorem on the dimension of the Gauss image for the maps $\gamma_{M}$ discussed above.

Remark 3.5. In more geometric language, a key point of our approach to Zak's theorem is to show that there is a non-zero global section of the sheaf $\omega_{X}(d-1)$. This should be compared to the work of Ein in [Ei], in which it is shown in the case where $X$ is smooth, that the global generation of $\omega_{X}(d-1)$ implies the finiteness of the Gauss map. Ein establishes the global generation of $\omega_{X}(d-1)$ in the smooth case using vanishing theorems in characteristic zero; the global generation of $\omega_{X}(d-1)$ in the prime characteristic case is proved in [S3]. This recovers the finiteness of the Gauss map in the smooth case in arbitrary characteristic.

\section{References}

[Ei] Ein, L., The ramification divisors for branched coverings of $\mathbb{P}_{k}^{n}$. Math. Ann. 261 (1982), 483-485.

[E] Elzein, F., Complexe dualisant et applications à la classe fondamentale d'un cycle. Bull. Soc. Math. France, Mémoire 58 (1978). 
[GH] Griffiths, P., Harris, J., Algebraic geometry and local differential geometry. Ann. Sci. École Norm. Sup. 12 (1979), 355-352.

[HH1] Hochster, M., Huneke, C., Tight closure, invariant theory, and the Briançon-Skoda theorem. J. Amer. Math. Soc. 3 (1990), 31-116.

[HH2] Hochster, M., Huneke, C., Infinite integral extensions and big Cohen-Macaulay algebras. Annals of Math. 135 (1992), 53-89.

[HH3] Hochster, M., Huneke, C., Tight closure in equal characteristic zero. preprint, 1999.

[KW] Kunz, E., Waldi, R., Regular differential forms. Contemp. Math. 79 (1988).

[L] Lipman, J., Dualizing sheaves, differentials and residues on algebraic varieties. Astérisque 117 (1984).

[M] McAdam, S., Asymptotic prime divisors. Springer Lecture Notes in Math. $\mathbf{1 0 2 3}$ (1983).

[S1] Smith, K.E., Tight closure and graded integral extensions. J. Algebra 175 (1995), 568-574.

[S2] Smith, K.E., Tight closure in graded rings. J. Math. Kyoto Univ. 37 (1997), 35-53.

[S3] Smith, K.E., A tight closure proof of Fujita's freeness conjecture for very ample line bundles. Math. Ann. 317 (2000), 285-293.

[SUV] Simis, A., Ulrich, B., Vasconcelos, W., Rees algebras of modules. preprint.

[Z] Zak, F.L., Tangents and secants of algebraic varieties. Transl. Math. Monogr. 127 (1993) Amer. Math. Soc., Providence, RI 